\title{
El desarrollo de las competencias emocionales en alumnado de secundaria: perfiles diferenciales en función del sexo
}

\section{Development of emotional competencies} in secondary school students: differential profiles by gender

\author{
Alicia Peñalva-Vélez*1 \\ alicia.penalva@unavarra.es \\ José-JAVIER LÓPEZ-GoÑI* \\ josejavier.lopez@unavarra.es \\ Maria-Isabel García-Manso** \\ misabelgmanso@hotmail.es \\ * Universidad Pública de Navarra, España \\ ** Valencian International University, España
}

\section{Resumen}

Se valoran las Habilidades Emocionales (HHEE) y el Bienestar Emocional (BE) de una muestra de 190 alumnos (97 hombres y 93 mujeres) de $4^{\circ}$ de ESO. Se valoró al alumnado en función de las HHEE y del $\mathrm{BE}$, y se realizó un análisis de clúster en función del BE. En líneas generales el alumnado presentaba unos buenos índices de HHEE y BE. No se encontraron diferencias estadísticamente significativas entre hombres y mujeres en ninguno de los dos. Sí se encontraron diferencias entre mujeres y hombres tanto en HHEE específicas como en componentes del BE. Entre el alumnado $(n=42)$ que se encon-

\begin{abstract}
:
This study evaluates Emotional Skills (E.S) and Emotional Wellness (E.W) of a sample of 190 4th of ESO students (97 men and 93 women). Students are assessed based on the E.S and E.W, and cluster analysis was performed based on the E.W. Overall the students had good levels of E.S and E.W. No statistically significant differences between men and women were found in the general index or the E.S and E.W, however differences were found in E.W and in specific components from E.S. Among students $(n=42)$ found a worse E.W most were women $(n=27,64.3 \%)$. Data from this study support the theory that the E.W
\end{abstract}

1 Dirección para correspondencia (correspondence address):

Alicia Peñalva-Vélez. Dpto. de Psicología y Pedagogía. Facultad de Ciencias Humanas y Sociales. Universidad Pública de Navarra. Campus Arrosadía, s/n. 31006 Pamplona (España). 
El desarrollo de las competencias emocionales en alumnado de secundaria: perfiles diferenciales en función del sexo

Alicia Peñalva-Vélez, José-Javier lópez-Goñi y Maria-Isabel García-Manso

tró con un peor BE la mayoría eran mujeres $(n=27 ; 64,3 \%)$. Los datos de este estudio avalan la teoría de que el BE se asienta sobre unas adecuadas HHEE. Se señala la evidencia de que existen diferencias de género en el desarrollo de las competencias emocionales de mujeres y hombres, y la necesidad de planificar una educación emocional en el marco del desarrollo curricular ordinario.

\section{Palabras clave:}

Educación emocional; diferencias de género; competencias emocionales; bienestar emocional; habilidades emocionales. sits on a E.S appropriate. The evidence indicates that there are gender differences in the development of emotional competencies of women and men, and the need to plan an emotional education within the regular curriculum development is noted.

\section{Keywords:}

Emotional education; emotional competences; emotional well-being; gender differences.

\section{Résumé:}

Dans cette étude on évalue les concepts "compétences émotionnelles" (HHEE) et "bienêtre émotionnel" (BE) d'un échantillon de 190 étudiants (97 hommes et 93 femmes). L'évaluation des élèves a été basée sur les HHEE et le BE, et un analyse de cluster a été réalisée sur la base du BE. Les élèves ont eu de bons taux des HHEE et du BE. On n'a pas trouvé aucune différence statistiquement significative entre les hommes et les femmes. Des différences entre les femmes et les hommes dans les HHEE, et dans des composants spécifiques du BE ont été trouvées. Les étudiants avec un pire $B E(n=42)$ étaient en majorité des femmes $(n=27 ; 64,3 \%)$. Les données de cette étude montrent que le développement du BE dépend d'un HHEE approprié. Il y a des différences entre les sexes quand on parle du développement des compétences émotionnelles des femmes et des hommes. Finalement la nécessité de planifier une éducation affective dans le développement du curriculum ordinaire est noté.

\section{Mots clés:}

Education emotionnelle; compétences émotionnelles; bien-être émotionnel; differences entre les sexes.

Fecha de recepción: 26-5-2015

Fecha de aceptación: 11-2-2016

\section{Introducción}

El concepto de competencia emocional hace referencia a los conocimientos, capacidades, habilidades y actitudes que se consideran necesarios para comprender, expresar y regular de forma apropiada los fenómenos emocionales (Bisquerra y Pérez-Escoda, 2007). Las competencias emocionales se consideran predictoras de un mejor afrontamiento ante los sucesos vitales cotidianos, y se relacionan además con mayores niveles de bienestar y ajuste psicológico en niños y adultos (Mayer, Salovey y Caruso, 2002; Pyhältö, Pietarinen y Salmela-Aro, 2011; Pena y Extreme- 
ra, 2012; Peñalva, López-Goñi y Landa, 2013; Salovey, Bedell, Detweiler y Mayer, 2000). También tienen una relación directa con el constructo de Inteligencia Emocional (I.E) (Agulló, 2011; Ballester-Vila y SánchezSantamaría, 2011; Bisquerra y Pérez-Escoda, 2007; Sánchez, FernándezBerrocal, Montañés y Latorre, 2008), definido como la habilidad para percibir, asimilar, comprender y regular las propias emociones (Martínez, Piqueras e Inglés, 2014).

El estudio de la I.E es relevante por sus implicaciones beneficiosas en la salud física y mental de las personas (Martínez et al., 2014; Martínez, Piqueras y Ramos, 2010). De hecho se considera que la I.E juega un papel muy importante en el autocontrol emocional y la capacidad adaptativa de los individuos para afrontar situaciones estresantes (Martínez et al., 2014).

Distintos estudios señalan la existencia de diferencias significativas en función del género en lo que se refiere al desarrollo de la I.E y por extensión de las competencias emocionales. Respecto a la I.E distintos autores informan de que tanto en la niñez como en la adolescencia, se constatan ya diferencias de género (Harrod y Scheer, 2005; Sánchez et al., 2008), y señalan una relación positiva y significativa entre género e I.E (Molero, Ortega y Moreno, 2010). Así lo explican Sánchez et al. (2008) cuando señalan que las diferencias de género encontradas en el ámbito de la I.E indican una mayor atención percibida hacia las propias emociones por parte de las mujeres (frente a los hombres). Mientras ellas se perciben más hábiles que los hombres a la hora de atender y comprender sus emociones, ellos se perciben más hábiles en relación con el control de impulsos y la tolerancia al estrés (Extremera, FernándezBerrocal y Salovey, 2006; Palomera, Gil-Olarte y Brackett, 2006; Young, 2006). En esta línea Mestre, Guil y Lim (2004) describen que cuando se analiza la inteligencia emocional desde la perspectiva de género, se observan rasgos o habilidades más desarrolladas en función del hecho de ser hombre o mujer.

Respecto a las competencias emocionales, los datos anteriores corroboran las diferencias de género que se muestran desde la niñez. Algunos autores atribuyen las diferencia de género a las diferentes pautas de socialización emocional que utilizan los padres en función del sexo (Eisenberg, Fabes y Spinrad, 2006; Martínez et al., 2014; Sánchez et al., 2008). Esta desigualdad en la educación emocional influiría en el desarrollo diferencial de las competencias emocionales en niños y niñas 
(Molero et al., 2010; Sánchez et al., 2008). Pena, Extremera y Rey (2011) relatan que las mujeres emplean en mayor medida estrategias basadas en la orientación negativa al problema, frente a los varones que se orientan al problema de forma más positiva. Señalan también que los varones muestran un estilo más impulsivo y recurren más a un estilo de evitación en la resolución de problemas que las mujeres. Otros autores como Bastian, Burns y Nettlebeck (2005), Extremera y Fernández-Berrocal (2005) o Salguero e Iruarrizaga (2006) afirman que las mujeres parecen informar de mayores niveles de atención a sus emociones que los varones. En definitiva, cada género parece presentar un perfil diferencial de puntos fuertes y débiles a nivel de competencia emocional (Caballero, 2004; Pena y Repetto, 2008).

Distintos autores afirman que el desarrollo de las competencias emocionales se logra básicamente a través de la educación emocional (Bisquerra, 2009; Durlak y Weisberg, 2010; Fernández-Abascal, 2008; Fernández-Berrocal y Ruiz-Aranda, 2008; Molero, 2009; Pena y Repetto, 2008; Sánchez, 2011). En la misma línea Félix-Mateo, Soriano-Ferrer, Godoy-Mesas y Martínez-Ruiz (2007) indican que la educación emocional es una forma de prevención primaria inespecífica, consistente en intentar minimizar la vulnerabilidad de las disfunciones o prevenir su ocurrencia. Para Salmurri y Skoknic (2005) y Salmurri (2012) la educación emocional tiene efectos cognitivos, conductuales y emocionales muy significativos en los alumnos, y entre sus objetivos principales se encuentran la promoción del bienestar personal y social de los sujetos. Los recursos y estrategias que proporciona la educación emocional son de tipo conductual, cognitivo, emocional, y de interacción social (Salmurri, 2004). La educación emocional tiene una función preventiva y de mejora de los estados emocionales, que se realiza a través del aprendizaje, el entrenamiento y la puesta en práctica de recursos y estrategias para amortiguar y minimizar las emociones excesivas o excesivamente negativas, y promover y aumentar las positivas (Salmurri, 2012).

Murillo y Hernández (2011) afirman que sin un plan explícito de intervención en educación emocional, los efectos de la escuela sobre el desarrollo de ciertas variables socio-afectivas como la autoestima son casi nulos. Sin embargo Pérez-González (2012) indica que en la mayoría de las escuelas no se dedica tiempo en la programación curricular semanal a actividades de educación emocional, algo que contrasta con los autores que en la actualidad insisten en los beneficios de la edu- 
cación emocional para el desarrollo de las competencias emocionales del alumnado (Bisquerra, 2008 y 2012; Carpena, 2010; Carpena, 2013; Extremera y Fernández-Berrocal, 2013; Royo, 2013; Salmurri, 2013; Salmurri, 2004 y Vallés, 2008). En este sentido estudios como los de Diekstra (2008), Durlak, Weissberg, Dymnicki, Taylor y Schellinger (2011) o Zins (2004), muestran evidencias empíricas acerca de la eficacia y beneficios de los programas de educación emocional. En esta misma línea Salmurri y Skoknic, (2005) indican que en una comunidad escolar, una intervención basada en la adquisición y entrenamiento de habilidades sociales, de interacción y de autocontrol emocional, podría mejorar la salud psicológica de profesores y alumnos.

Este estudio busca establecer el nivel de habilidades emocionales y el índice general de bienestar que presenta una muestra de 190 alumnos y alumnas de $4^{\circ}$ de ESO pertenecientes a un instituto público de educación secundaria de la provincia de Badajoz. El instituto se caracteriza por no contemplar, en el momento de la pasación de los cuestionarios, la formación a nivel emocional de su alumnado. Los objetivos que se plantean por lo tanto son: (1) valorar las Habilidades Emocionales del alumnado de Secundaria y las diferencias entre hombres y mujeres; (2) valorar el Índice General de Bienestar y sus diferencias entre sexos; (3) identificar al alumnado con mayor déficit en Índice General de Bienestar; y (4) determinar si existe relación entre el mayor déficit en el Índice General de Bienestar y las Habilidades Emocionales.

\section{Metodología}

\section{Participantes}

La muestra está compuesta por un total de 190 alumnos del IES Donoso Cortés de Don Benito (Badajoz). El centro es de titularidad pública, y el alumnado pertenece a los cursos de $4^{\circ}$ de ESO. En la Tabla 1 se presenta la distribución de la muestra por sexo y edad. No se aprecian diferencias estadísticamente significativas en estas dos variables. 
Tabla 1. Distribución por sexo y edad de la muestra.

\begin{tabular}{lccccccc}
\hline & \multicolumn{2}{c}{$\begin{array}{c}\text { Total } \\
(\mathrm{N}=190)\end{array}$} & \multicolumn{2}{c}{$\begin{array}{c}\text { Hombres } \\
(\mathrm{n}=97)\end{array}$} & \multicolumn{2}{c}{$\begin{array}{c}\text { Mujeres } \\
(\mathrm{n}=93)\end{array}$} & \\
\hline Edad & $\mathrm{N}$ & $(\%)$ & $\mathrm{n}$ & $(\%)$ & $\mathrm{n}$ & $(\%)$ & $X^{2}$ (g.l.) \\
\hline 14 & 66 & $34.7 \%$ & 37 & $38.1 \%$ & 29 & $31.2 \%$ & \\
15 & 67 & $35.3 \%$ & 34 & $35.1 \%$ & 33 & $35.5 \%$ & $2,3(3)$ \\
16 & 47 & $24.7 \%$ & 20 & $20.6 \%$ & 27 & $29.0 \%$ & \\
17 & 10 & $5.3 \%$ & 6 & $6.2 \%$ & 4 & $4.3 \%$ & \\
\hline
\end{tabular}

Fuente: elaboración propia.

\section{Instrumentos de evaluación}

La Escala de Habilidades Emocionales (EHE-1) (Salmurri, 2012) consta de 64 preguntas (38 con connotaciones negativas y 26 con connotaciones positivas). Las preguntas tienen como respuesta: nunca (1), casi nunca (2), algunas veces (3), bastantes veces (4), casi siempre (5) y siempre (6). De este cuestionario se puede obtener una puntuación global, aunque también es muy interesante el análisis individualizado de los ítems. El alfa de Cronbach obtenido en esta muestra fue de 0,784.

El Índice General de Bienestar Emocional (IGBE) (Salmurri, 2009) está formado por cinco preguntas que hacen referencia a: autoestima, autocontrol de conducta, autocontrol emocional, estilo cognitivo positivo y relaciones con los otros y se evalúan de 0 a 10 . Para ello se pide a los alumnos que valoren sus experiencias en los últimos 1-2 meses para poder responder. El alfa de Cronbach de esta muestra fue de 0,82.

\section{Procedimiento}

Tras contactar con el Equipo Directivo del Centro se procedió a la pasación de los cuestionarios en cada una de las aulas. Los instrumentos fueron aplicados por los tutores de cada aula a todo el alumnado. En todo momento se enfatizó el anonimato y la confidencialidad de los resultados, así como la voluntariedad de participar en el estudio. Los investigadores se aseguraron a través de la orientadora del centro de transmitir a todos los profesores las instrucciones específicas relativas a la aplicación de ambas pruebas. 


\section{Análisis estadístico}

En primer lugar se realizó un análisis univariado de los diferentes ítems de los dos cuestionarios empleados (EHE-I e IGBE). Más concretamente, se obtuvieron las frecuencias obtenidas en cada pregunta, así como el tanto por ciento de cada una de las posibles respuestas. También se calcularon diferentes estadísticos descriptivos tales como la media, mediana, desviación típica, valores máximo y mínimo de cada ítem y las puntuaciones deciles para las escalas globales. A continuación se realizó un análisis de la fiabilidad de la EHE-I.

Con el fin de determinar la aleatoridad de los resultados de la muestra se realizó la prueba de rachas para todas y cada una de las variables empleadas. Dicho test se realizó en dos ocasiones, estableciendo como punto de corte la mediana y la media respectivamente. El resultado de dichas pruebas confirmó la aleatoriedad de los datos. Este hecho, unido al alto tamaño muestral con el que se contaba $(n>40)$ determinó que se optase por emplear estadística paramétrica.

A continuación se compararon los resultados obtenidos en la EHE-I entre hombres y mujeres. Para la comparación de medias se utilizó la prueba $t$ de Student para grupos independientes. En función de la prueba de homogeneidad de varianzas (prueba de Levéne) se empleó o no la corrección correspondiente. En todos los casos se consideró como estadísticamente significativa una $p<0,05$.

En cuanto al IGBE, tras el análisis univariado de los ítems y del factor general, también se procedió a una comparación entre hombres y mujeres, aunque en este caso únicamente con la prueba $t$ de Student para grupos independientes.

Con el objetivo de clasificar al alumnado en función del IGBE, se procedió finalmente a realizar un análisis de clúster a partir de los ítems del IGBE. Ésta es una estrategia que ha sido empleada con éxito en otros ámbitos de investigación (López-Goñi, Fernández-Montalvo y Arteaga, 2012; Peñalva, López-Goñi y Landa, 2013). No se incluyó el índice general porque es la suma de los ítems individuales y resultaría redundante. El análisis resultante obtuvo dos grupos de alumnos. A partir de este análisis de clúster se procedió a comparar los sujetos de ambos grupos en todas las variables incluidas en el estudio. De esta manera se identificó un grupo de alumnos con bajas habilidades emocionales, respecto de otro grupo de alumnos con altas habilidades. 
Todos los cálculos estadísticos se realizaron con el programa SPSS (vs. 15.0).

\section{Resultados}

\section{Resultados en la EHE-I y comparación entre sexos}

En la Tabla 2 se muestran los datos descriptivos para el índice general de la EHE-I. No se aprecian diferencias estadísticamente significativas en la media global entre hombres y mujeres $(t=0,30 ;$ g.l. $=181,46 ; p$ $=0,764)$.

Tabla 2. Descriptivos para el índice general en las Escala de Habilidades Emocionales y comparación hombres-mujeres.

\begin{tabular}{|c|c|c|c|c|}
\hline & & $\begin{array}{c}\text { Total } \\
(\mathrm{N}=190)\end{array}$ & $\begin{array}{l}\text { Hombres } \\
(\mathrm{N}=97)\end{array}$ & $\begin{array}{l}\text { Mujeres } \\
(N=93)\end{array}$ \\
\hline Media & & 281.77 & 282.51 & 281.11 \\
\hline Mediana & & 286.00 & 292.00 & 283.00 \\
\hline Desviación típica & & 34.378 & 38.174 & 30.231 \\
\hline Valor mínimo & & 192 & 192 & 217 \\
\hline Valor máximo & & 352 & 352 & 352 \\
\hline \multirow{9}{*}{ Percentiles } & 10 & 232.10 & 224.60 & 238.80 \\
\hline & 20 & 249.00 & 244.60 & 249.00 \\
\hline & 30 & 265.00 & 267.60 & 262.40 \\
\hline & 40 & 275.00 & 277.00 & 275.00 \\
\hline & 50 & 286.00 & 292.00 & 283.00 \\
\hline & 60 & 296.60 & 300.80 & 294.00 \\
\hline & 70 & 304.00 & 307.60 & 298.80 \\
\hline & 80 & 311.80 & 314.40 & 306.60 \\
\hline & 90 & 323.00 & 326.00 & 321.40 \\
\hline
\end{tabular}

Fuente: elaboración propia.

Cuando se analizan los ítems individualmente existen diferencias estadísticamente significativas entre hombres y mujeres en 14 de los 64 ítems (Tabla 3). En este sentido los adolescentes tienden a sentirse menos 
tensos o ansiosos que las adolescentes, y a preocuparse menos que ellas por las pequeñas cosas. Los sentimientos de insatisfacción son también menores en el caso de los adolescentes. Respecto a la atención que se presta a los demás cuando hablan, los adolescentes parecen mostrarse más atentos por lo general que las adolescentes. Pasa lo mismo en cuanto al control de los estados de ánimo, cuando los adolescentes demuestran más capacidad para encontrar formas de sentirse mejor que las adolescentes. Los adolescentes tienen más claros los objetivos básicos de su vida, y saben mejor que las adolescentes qué hacer cuando se sienten mal.

Si se analizan las diferencias de las adolescentes con respecto a los adolescentes, se ve que éstas se sitúan mejor en aspectos como la capacidad de empatía: perciben cuando una persona está triste, o enfadada, y tienen en cuenta los sentimientos de los otros. Expresan con más facilidad que los adolescentes sus sentimientos, pero se muestran excesivamente preocupadas por pequeños detalles, y se culpabilizan a sí mismas cuando se sienten mal por algo. Son más inseguras que los adolescentes, pero buscan más ayuda que ellos para resolver dificultades. Los sentimientos de inferioridad, tristeza o desánimo son mayores en las adolescentes que en los adolescentes.

Tabla 3. Descriptivos respuestas en las Escalas de Habilidades Emocionales ordenadas según media (descendente) y comparación hombres - mujeres.

\begin{tabular}{|c|c|c|c|c|c|c|c|c|c|}
\hline & $\begin{array}{r}\mathrm{To} \\
(\mathrm{N}=\end{array}$ & 190) & $\begin{array}{l}\text { Hom } \\
(\mathrm{n}=\end{array}$ & $\begin{array}{l}\text { abres } \\
97)\end{array}$ & & $\begin{array}{l}\text { jeres } \\
=93) \\
\end{array}$ & & & \\
\hline & M & (D.T). & $M$ & (D.T.) & $M$ & (D.T.) & $t$ & g.l. & Sig. \\
\hline $\begin{array}{l}\text { Me siento bien en compañía } \\
\text { de otras personas }\end{array}$ & 5.24 & 1.00 & 5.11 & 1.12 & 5.38 & 0.85 & -1.86 & 178.70 & 0.065 \\
\hline $\begin{array}{l}\text { Me doy cuenta cuando una } \\
\text { persona se siente feliz }\end{array}$ & 5.06 & 1.01 & 4.92 & 1.11 & 5.20 & 0.89 & -1.96 & 188.00 & 0.051 \\
\hline $\begin{array}{l}\text { Me doy cuenta cuando una } \\
\text { persona se siente triste } \\
\text { Me disculpo o pido perdón }\end{array}$ & 4.94 & 1.08 & 4.77 & 1.13 & 5.11 & 1.01 & -2.16 & 187.00 & 0.032 \\
\hline cuando me equivoco & 4.88 & 1.32 & 4.76 & 1.29 & 5.00 & 1.35 & -1.23 & 187.00 & 0.218 \\
\hline $\begin{array}{l}\text { Me doy cuenta cuando una } \\
\text { persona se siente enfadada }\end{array}$ & 4.87 & 1.09 & 4.70 & 1.13 & 5.05 & 1.03 & -2.26 & 187.46 & 0.025 \\
\hline $\begin{array}{l}\text { Soy respetuoso/a y } \\
\text { considerado/a con los demás }\end{array}$ & 4.72 & 1.22 & 4.57 & 1.23 & 4.88 & 1.20 & -1.75 & 187.00 & 0.082 \\
\hline $\begin{array}{l}\text { Me comporto con honestidad } \\
\text { y honradez }\end{array}$ & 4.63 & 1.12 & 4.56 & 1.22 & 4.71 & 1.00 & -0.89 & 181.66 & 0.377 \\
\hline
\end{tabular}


El desarrollo de las competencias emocionales en alumnado de secundaria: perfiles diferenciales en función del sexo

Alicia Peñalva-Vélez, José-Javier lópez-Goñi y Maria-Isabel García-Manso

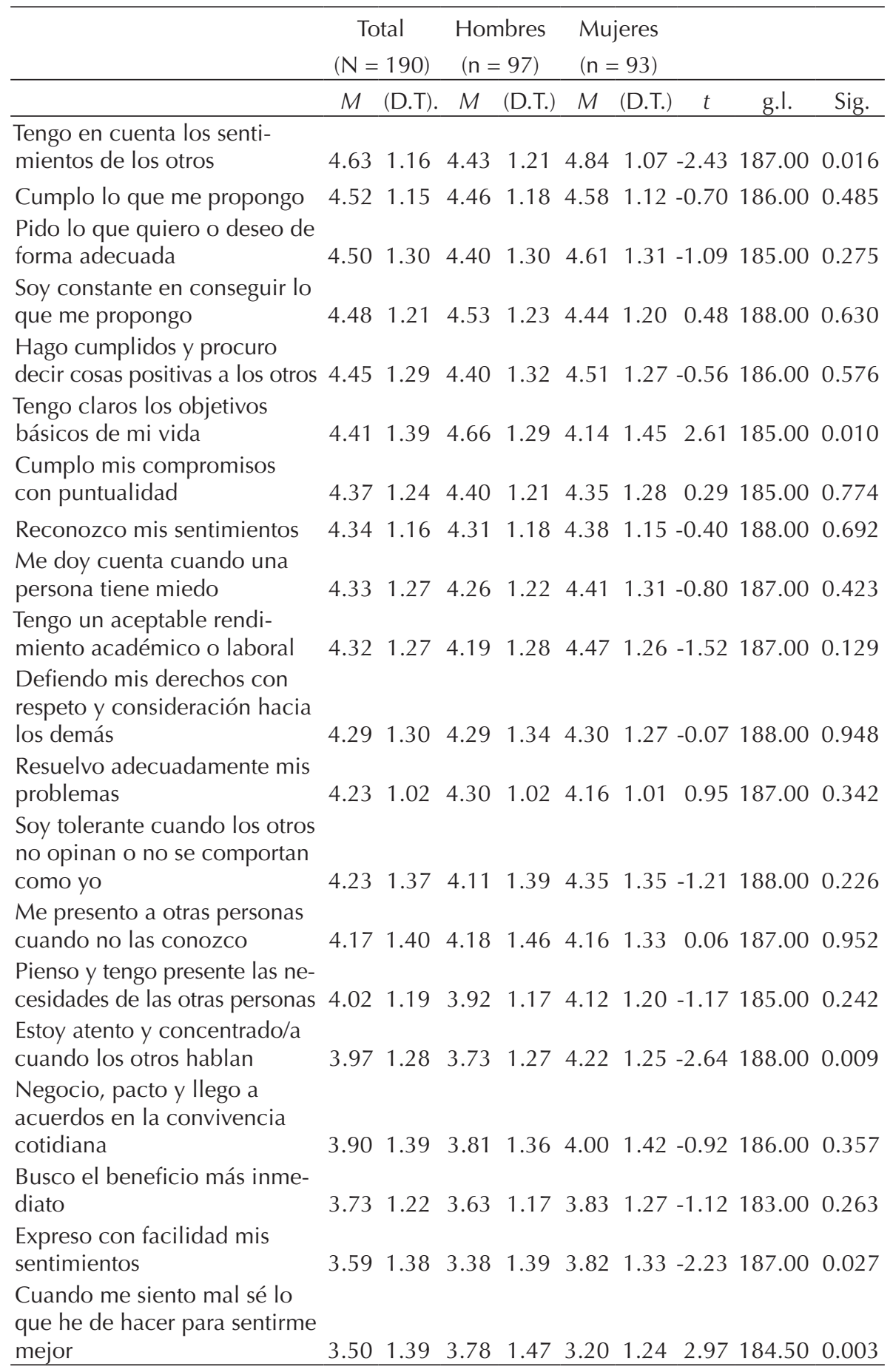


El desarrollo de las competencias emocionales en alumnado de secundaria: perfiles diferenciales en función del sexo

Alicia Peñalva-Vélez, José-Javier López-Goñi y Maria-Isabel García-Manso

\begin{tabular}{|c|c|c|c|c|c|c|c|c|c|}
\hline & \multicolumn{2}{|c|}{$\begin{array}{c}\text { Total } \\
(\mathrm{N}=190)\end{array}$} & \multicolumn{2}{|c|}{$\begin{array}{c}\text { Hombres } \\
(\mathrm{n}=97)\end{array}$} & \multicolumn{2}{|c|}{$\begin{array}{l}\text { Mujeres } \\
(\mathrm{n}=93)\end{array}$} & & \multirow[b]{2}{*}{ g.l. } & \multirow[b]{2}{*}{ Sig. } \\
\hline & $M$ & (D.T). & . $M$ & (D.T.) & $M$ & (D.T.) & & & \\
\hline $\begin{array}{l}\text { Me cuesta pedir ayuda aun- } \\
\text { que lo necesite }\end{array}$ & 3.32 & 1.33 & 3.33 & 1.27 & 3.30 & 1.40 & 0.13 & 184.00 & 0.897 \\
\hline $\begin{array}{l}\text { Me preocupo excesivamente } \\
\text { por las pequeñas cosas }\end{array}$ & 3.31 & 1.45 & 2.91 & 1.32 & 3.72 & 1.47 & -4.01 & 183.73 & 0.000 \\
\hline Me cuesta esforzarme & 3.19 & 1.18 & 3.29 & 1.27 & 3.08 & 1.08 & 1.24 & 185.01 & 0.215 \\
\hline $\begin{array}{l}\text { Cuando me siento mal pienso } \\
\text { que es culpa mía }\end{array}$ & 3.12 & 1.23 & 2.90 & 1.19 & 3.35 & 1.25 & -2.59 & 188.00 & 0.010 \\
\hline Tengo sentimientos de culpa & 3.12 & 1.40 & 3.07 & 1.38 & 3.16 & 1.42 & -0.44 & 188.00 & 0.662 \\
\hline $\begin{array}{l}\text { Me siento tímido/a o } \\
\text { reservado/a cuando viene } \\
\text { algún extraño a casa }\end{array}$ & 3.02 & 1.58 & 2.88 & 1.55 & 3.17 & 1.60 & -1.30 & 187.00 & 0.196 \\
\hline $\begin{array}{l}\text { Soy rencoroso/a y pienso en } \\
\text { vengarme aunque luego no } \\
\text { lo haga }\end{array}$ & 3.02 & 1.58 & 2.91 & 1.46 & 3.13 & 1.70 & -0.96 & 181.11 & 0.337 \\
\hline En casa me enfado & 2.99 & 1.15 & 2.75 & 1.15 & 3.25 & 1.11 & -3.02 & 188.00 & 0.003 \\
\hline Me cuesta aceptar mis errores & 2.82 & 1.31 & 2.91 & 1.43 & 2.74 & 1.19 & 0.85 & 186.00 & 0.396 \\
\hline $\begin{array}{l}\text { Reacciono desproporcionada- } \\
\text { mente ante una negativa }\end{array}$ & 2.79 & 1.17 & 2.79 & 1.12 & 2.79 & 1.23 & 0.00 & 183.00 & 0.997 \\
\hline Soy celoso/a & 2.77 & 1.46 & 2.59 & 1.35 & 2.96 & 1.56 & -1.71 & 187.00 & 0.088 \\
\hline $\begin{array}{l}\text { Me cuesta aceptar opiniones } \\
\text { diferentes de las mías }\end{array}$ & 2.70 & 1.02 & 2.76 & 0.90 & 2.63 & 1.13 & 0.86 & 175.52 & 0.388 \\
\hline Me siento insatisfecho/a & 2.57 & 1.30 & 2.49 & 1.23 & 2.66 & 1.37 & -0.88 & 187.00 & 0.381 \\
\hline $\begin{array}{l}\text { Me cuesta adaptarme a } \\
\text { situaciones nuevas o poco } \\
\text { habituales }\end{array}$ & 2.57 & 1.09 & 2.60 & 1.18 & 2.54 & 1.00 & 0.39 & 182.05 & 0.695 \\
\hline $\begin{array}{l}\text { Siento miedo ante cualquier } \\
\text { acontecimiento o situación } \\
\text { nueva }\end{array}$ & 2.57 & 1.07 & 2.36 & 1.03 & 2.78 & 1.07 & -2.78 & 188.00 & 0.006 \\
\hline $\begin{array}{l}\text { Provoco a mis hermanos o a } \\
\text { mis padres o a mi pareja una } \\
\text { vez o más por semana }\end{array}$ & 2.56 & 1.47 & 2.58 & 1.55 & 2.53 & 1.39 & 0.26 & 187.00 & 0.792 \\
\hline Me siento inseguro/a & 2.51 & 1.39 & 2.27 & 1.34 & 2.75 & 1.41 & -2.41 & 187.00 & 0.017 \\
\hline $\begin{array}{l}\text { Busco ayuda ante las más } \\
\text { pequeñas dificultades }\end{array}$ & 2.50 & 1.09 & 2.32 & 1.07 & 2.68 & 1.10 & -2.25 & 187.00 & 0.026 \\
\hline $\begin{array}{l}\text { Tengo una opinión poco po- } \\
\text { sitiva de las personas de fuera } \\
\text { de casa }\end{array}$ & 2.47 & 1.37 & 2.55 & 1.39 & 2.39 & 1.34 & 0.81 & 186.00 & 0.421 \\
\hline $\begin{array}{l}\text { Me siento desbordado/a y } \\
\text { dominado/a por la rabia }\end{array}$ & 2.47 & 1.19 & 2.52 & 1.23 & 2.42 & 1.15 & 0.56 & 188.00 & 0.579 \\
\hline $\begin{array}{l}\text { Estoy apático/a o } \\
\text { desmotivado/a }\end{array}$ & 2.43 & 1.06 & 2.35 & 1.10 & 2.51 & 1.02 & -0.99 & 183.00 & 0.324 \\
\hline
\end{tabular}


El desarrollo de las competencias emocionales en alumnado de secundaria: perfiles diferenciales en función del sexo

Alicia Peñalva-Vélez, José-Javier lópez-Goñi y Maria-Isabel García-Manso

\begin{tabular}{|c|c|c|c|c|c|c|c|c|c|}
\hline & $\begin{array}{r}\text { Tot } \\
(\mathrm{N}=\end{array}$ & 190) & $\begin{array}{l}\text { Hom } \\
(\mathrm{n}=\end{array}$ & $\begin{array}{l}\text { abres } \\
=97)\end{array}$ & $\begin{array}{l}\text { Muj } \\
(\mathrm{n}=\end{array}$ & $\begin{array}{l}\text { jeres } \\
=93) \\
\end{array}$ & & & \\
\hline & $M$ & (D.T). & $M$ & (D.T.) & $M$ & (D.T.) & $t$ & g.l. & Sig. \\
\hline Consumo alcohol & 2.42 & 1.51 & 2.44 & 1.59 & 2.39 & 1.44 & 0.24 & 187.00 & 0.814 \\
\hline $\begin{array}{l}\text { Tengo una visión poco positi- } \\
\text { va de mi futuro }\end{array}$ & 2.40 & 1.55 & 2.16 & 1.45 & 2.63 & 1.62 & -2.10 & 184.00 & 0.037 \\
\hline $\begin{array}{l}\text { Estoy desilusionado/a o } \\
\text { desanimado/a }\end{array}$ & 2.37 & 1.26 & 2.17 & 1.21 & 2.58 & 1.29 & -2.28 & 187.00 & 0.024 \\
\hline Estoy triste & 2.33 & 1.14 & 2.16 & 1.12 & 2.51 & 1.14 & -2.07 & 188.00 & 0.039 \\
\hline $\begin{array}{l}\text { Cuando me siento mal pienso } \\
\text { que es culpa de los demás }\end{array}$ & 2.31 & 1.13 & 2.39 & 1.16 & 2.24 & 1.11 & 0.88 & 186.00 & 0.378 \\
\hline
\end{tabular}

Fuente: elaboración propia.

\section{Resultados en el IGBE y comparación entre sexos}

En cuanto al IGBE, de los 5 ítems que componen la escala, se observan diferencias estadísticamente significativas entre ambos sexos en dos de ellos: el autocontrol emocional y el estilo cognitivo (Tabla 4). En ambas los adolescentes puntúan más alto que las adolescentes. Se puede destacar que en los 3 ítems restantes los adolescentes puntúan más alto que las adolescentes en autoestima y autocontrol de la conducta, pero más bajo que ellas en interacción social.

Tabla 4. Resultados descriptivos del Índice General de Bienestar Emocional y comparación hombres-mujeres.

\begin{tabular}{|c|c|c|c|c|c|c|c|c|c|}
\hline & \multicolumn{2}{|c|}{$\begin{array}{c}\text { Total } \\
(\mathrm{n}=190)\end{array}$} & \multicolumn{2}{|c|}{$\begin{array}{c}\text { Hombres } \\
(\mathrm{n}=97)\end{array}$} & \multicolumn{2}{|c|}{$\begin{array}{l}\text { Mujeres } \\
(\mathrm{n}=93)\end{array}$} & & \multirow[b]{2}{*}{ g.l. } & \multirow[b]{2}{*}{ sig. } \\
\hline & M & (D.T.) & M & (D.T.) & $M$ & (D.T.) & & & \\
\hline Autoestima & 7.26 & (1.91) & 7.42 & $(1.8)$ & 7.09 & $(2.02)$ & 1.2 & 188 & 0.232 \\
\hline $\begin{array}{l}\text { Autocontrol } \\
\text { conducta }\end{array}$ & 7.61 & $(1.60)$ & 7.68 & (1.7) & 7.54 & (1.5) & 0.57 & 188 & 0.571 \\
\hline $\begin{array}{l}\text { Autocontrol } \\
\text { emocional }\end{array}$ & 7.17 & (2.05) & 7.54 & $(1.9)$ & 6.79 & $(2.17)$ & 2.55 & 188 & 0.011 \\
\hline Estilo cognitivo & 7.34 & (1.91) & 7.61 & $(1.9)$ & 7.06 & (1.91) & 2.0 & 188 & 0.048 \\
\hline Interacción social & 8.25 & $(1.95)$ & 8.2 & $(2.1)$ & 8.31 & $(1.78)$ & -0.37 & 188 & 0.710 \\
\hline Total & 38.04 & $(9.24)$ & 39.24 & (11.0) & 36.79 & $(7.13)$ & 1.84 & 188 & 0.068 \\
\hline
\end{tabular}

Fuente: elaboración propia. 


\section{Análisis de clúster y caracterización de los grupos resultantes}

Un $77,9 \%$ de los sujetos $(n=148)$ fue asignado al clúster 1 , y un $22,1 \%$ $(\mathrm{n}=42)$ fue asignado al clúster 2 . En la (Tabla 5$)$ se presentan los resultados de la comparación entre los sujetos de ambos grupos.

Tabla 5. Comparación de medias en edad, índice total EHE-I y en los ítems del cuestionario del Índice General de Bienestar Emocional entre el clúster 1 y clúster 2.

$$
\begin{array}{cc}
\text { Clúster } 1 & \text { Clúster } 2 \\
(\mathrm{n}=148) & (\mathrm{n}=42)
\end{array}
$$

\begin{tabular}{|c|c|c|c|c|c|c|c|c|}
\hline \multicolumn{9}{|l|}{ Sexo } \\
\hline Hombres & 82 & (55.4\%) & 15 & $(35.7 \%)$ & & $5.1^{*}$ & 1 & \\
\hline \multirow[t]{2}{*}{ Mujeres } & 66 & $(44.6 \%)$ & 27 & $(64.3 \%)$ & & & & \\
\hline & $M$ & (D.T.) & $M$ & (D.T.) & Dif. & $t$ & g.I. & Sign. \\
\hline Edad & 14.91 & $(0.84)$ & 15.36 & $(1.03)$ & -0.45 & -2.61 & 57.1 & 0.012 \\
\hline Índice Total EHE-I & 290.62 & $(29.86)$ & 250.57 & $(31.15)$ & 40.05 & 7.60 & 188.0 & 0.000 \\
\hline \multicolumn{9}{|c|}{ Índice General Bienestar Emocional } \\
\hline Autoestima & 7.97 & $(1.22)$ & 4.75 & $(1.76)$ & 3.22 & 11.15 & 52.7 & 0.000 \\
\hline $\begin{array}{l}\text { Autocontrol de } \\
\text { conducta }\end{array}$ & 8.01 & $(1.39)$ & 6.21 & $(1.54)$ & 1.79 & 7.21 & 188.0 & 0.000 \\
\hline $\begin{array}{l}\text { Autocontrol } \\
\text { emocional }\end{array}$ & 7.89 & $(1.34)$ & 4.64 & $(2.12)$ & 3.25 & 9.42 & 50.7 & 0.000 \\
\hline Estilo cognitivo & 7.94 & $(1.29)$ & 5.24 & $(2.26)$ & 2.70 & 7.40 & 48.8 & 0.000 \\
\hline Interacción social & 8.97 & (1.00) & 5.74 & $(2.38)$ & 3.23 & 8.58 & 45.2 & 0.000 \\
\hline Índice Total & 41.29 & $(7.40)$ & 26.58 & $(4.98)$ & 14.71 & 12.12 & 188.0 & 0.000 \\
\hline
\end{tabular}

Los sujetos del clúster 2 presentan un mayor porcentaje de mujeres, y una edad media mayor que los sujetos del clúster 1 . En el resto de variables analizadas, los sujetos del clúster 2 presentan unos resultados menores que los sujetos del clúster 1.

\section{Discusión y conclusiones}

En líneas generales, el alumnado de la muestra presenta unas adecuadas Habilidades Emocionales, así como un aceptable grado de Bienestar Emocional. Ahora bien, los resultados de este estudio muestran diferencias de género en las competencias emocionales del alumnado. Las ado- 
lescentes de la muestra presentan buenas habilidades sociales básicas, pero peores habilidades en conciencia emocional, regulación emocional y autonomía emocional. En concreto, las adolescentes captan mejor el clima emocional del contexto, expresan con más facilidad sus sentimientos, pero tienen menos conciencia sobre sus propias emociones. Se culpabilizan a sí mismas cuando se sienten mal por algo y son más inseguras que los adolescentes. Además, los sentimientos de inferioridad, tristeza o desánimo son mayores. Es decir, las adolescentes presentan una menor regulación emocional y menor capacidad de afrontamiento de emociones negativas.

Los adolescentes de la muestra, por su parte, tienen una mayor conciencia emocional (son más conscientes de sus propias emociones y más capaces de identificar de manera adecuada las distintas emociones); y muestran una mayor regulación emocional (capacidad para manejar sus emociones de manera adecuada) siendo más hábiles en el afrontamiento de las emociones negativas. Como consecuencia, los adolescentes presentan una mayor autonomía emocional que se concreta en una mayor autoestima y una actitud más positiva ante la vida. Siguiendo a Bisquerra y Pérez-Escoda (2007), se puede afirmar que los adolescentes de la muestra presentan mayores competencias para la vida y el bienestar que las adolescentes de la misma. Esto conlleva tener capacidad para adoptar comportamientos apropiados y responsables en el sentido de saber fijar objetivos adaptativos, o tomar decisiones de forma fundamentada. Otros autores también han encontrado resultados muy similares en diferentes muestras de adolescentes y con distintos instrumentos de evaluación (Caballero, 2004; Pena, Extremera y Rey, 2011, Pena y Repetto, 2008). Martínez et al. (2014) indican que aunque las mujeres presentan niveles más altos de atención hacia las emociones que los hombres, esto se relaciona también con estrategias de afrontamiento inadecuadas. Los hombres presentan así una mayor capacidad de autorregulación ante situaciones de estrés.

A partir del IGBE se identificaron dos grupos de alumnos. Aquellos que presentaban unas peores puntuaciones en Bienestar Emocional (el $22,1 \%$ de la muestra), presentaban, asimismo, unas peores habilidades emocionales en el EHE-I. Estos datos avalan la teoría de que el bienestar emocional se asienta, básicamente en unas adecuadas habilidades emocionales. Además, el grupo con peor Bienestar Emocional estaba compuesto en mayor proporción por mujeres (64,3\% frente a $44,6 \%$ ) y 
tenían una edad media mayor que los sujetos del grupo con mayor bienestar. Nuevamente estos datos, sustentan la hipótesis de que las mujeres tienen peores habilidades en conciencia emocional, regulación emocional y autonomía emocional. Así pues, cada género parece presentar perfiles diferenciales de puntos fuertes y débiles en lo que a competencias emocionales se refiere (Pena y Repetto, 2008).

Parece necesario planificar en el marco del desarrollo curricular ordinario la educación emocional como vía de formación en competencias emocionales. Una educación que debe centrarse en el desarrollo de competencias emocionales como: el conocimiento de las propias emociones y las emociones de los demás, la regulación emocional, el control de los impulsos, la tolerancia a la frustración, el desarrollo de la autoestima y la automotivación, o el establecimiento de relaciones interpersonales positivas (Bisquerra, 2002 y 2003; Bisquerra y Pérez-Escoda, 2007; Giménez y Quintanilla, 2009; Salmurri, 2013). Una educación que debe además tener en cuenta las diferencias de género que se observan en este y otros estudios ya mencionados.

La finalidad de la educación emocional no es otra que dotar al individuo de recursos y estrategias que le permitan tener un mayor control de la presión interna y externa (Salmurri, 2004; Salmurri, 2013). Tiene una función preventiva, de mejora de los estados emocionales, que realiza a través del aprendizaje, el entrenamiento y la puesta en práctica de recursos y estrategias para: (1) amortiguar y minimizar las emociones excesivas o excesivamente negativas, (2) promover y aumentar las positivas. Debe contemplar necesariamente las evidencias que se presentan en este estudio respecto al desarrollo diferencial de las competencias emocionales en niños y niñas, incidiendo en función de cada sexo en los puntos fuertes y débiles que cada uno presenta a nivel de competencia emocional (Caballero, 2004; Pena y Repetto, 2008; Salmurri, 2009, 2012 y 2013).

Tal y como señala Carpena (2010 y 2013), la educación emocional se orienta a que el alumnado adquiera conocimientos fundamentados sobre las emociones, para que sea capaz de valorar las suyas y las de los demás. Trata igualmente de que adquiera cierto grado de competencia en su regulación, así como de que desarrolle unas habilidades sociales fundamentales para el desarrollo integral de las personas, tanto a nivel social como emocional. Los estados emocionales tienen una relación directa con los aprendizajes, y la falta de habilidad para regular las 
emociones puede suponer graves trastornos personales, interpersonales y/o académicos, sobre todo con respecto a las emociones perturbadoras (Martínez, Piqueras y Ramos, 2010; Martins, Ramalho y Morin, 2010). Por todo ello, tal y como afirma Sánchez (2010), es necesario reforzar la dimensión emocional y social, junto con la cognitiva, en el proceso de aprendizaje de los alumnos. Porque además, y finalmente, las emociones tienen gran influencia en la motivación, y son esenciales para la gestión del conflicto y la convivencia escolar (Bisquerra, 2008; FernándezBerrocal y Ruiz-Aranda, 2008; Palomera, 2008).

Este estudio presenta algunas limitaciones, por lo que se debe ser cauteloso en la generalización de sus resultados. Es un estudio que se ha basado únicamente en la cumplimentación de dos cuestionarios por parte del alumnado. Si bien es cierto que ésta es una práctica habitual, también es cierto que existe una diferencia entre la autopercepción de las competencias y la auténtica competencia. Es relativamente habitual que personas competentes se crean incompetentes, y en unos pocos casos que personas incompetentes se sientan competentes. Por ello una sugerente línea de investigación futura será validar los resultados de esta investigación con comprobaciones de la competencia emocional incorporando por ejemplo, entrevistas en profundidad, o role-playing. Por otro lado, sería deseable poder comparar los resultados de este alumnado con los de otro centro que sí disponga de programas específicos de educación emocional. De esta manera se podrá valorar la eficacia de este tipo de programas.

\section{Referencias bibliográficas}

AGULLÓ, M.J. (2011). Evaluación de la educación emocional en el ciclo medio de educación primaria. Revista de Educación, 354, 765-783.

BALLESTER-VILA, M.G. y SÁNCHEZ-SANTAMARÍA, J. (2012). La dimensión pedagógica del enfoque de competencias en educación obligatoria. Ensayos, Revista de la Facultad de Educación de Albacete, 26, 17-34.

BASTIAN, V., BURNS, N. y NETTLEBECK, T. (2005). Emotional intelligence predicts life skills, but not as well as personality and cognitive abilities. Personality and Individual Differences, 39, 1135-1145.

BISQUERRA, R (2002). La competencia emocional. En Álvarez, M y Bisquerra, R., Manual de Orientación y Tutoría (69-83). Barcelona: Praxis

BISQUERRA, R. (2003). Educación emocional y competencias básicas para la vida. Revista de Investigación Educativa, 21(1), 7-43. 
El desarrollo de las competencias emocionales en alumnado de secundaria: perfiles diferenciales en función del sexo Alicia Peñalva-Vélez, José-Javier López-Goñi y Maria-Isabel García-Manso

BISQUERRA, R. (2008). Educación para la ciudadanía y convivencia. El enfoque de la educación emocional. Bilbao: Praxis.

BISQUERRA, R. (2009). Psicopedagogía de las emociones. Madrid: Editorial Síntesis.

BISQUERRA, R. (2011). Diversidad y escuela inclusiva desde la educación emocional. En Navarro, J. (2011), Diversidad, Calidad y Equidad Educativa (pp.1-10). Murcia: Consejería de Educación, Formación y Empleo.

BISQUERRA, R. (Coord.) (2012).¿Cómo educar las emociones? La inteligencia emocional en la infancia y la adolescencia. Esplugues de Llobregat, Barcelona: Hospital Sant Joan de Déu.

BISQUERRA, R. y PÉREZ-ESCODA, N. (2007). Las competencias emocionales. Revista de Educación Siglo XXI, 10, 61-82.

CABALLERO, A. (2004). Cómo enfocar la educación emocional dentro del aula desde la perspectiva de género. En AA.VV., Actas de la Conferencia Internacional Orientación, Inclusión social y Desarrollo de la Carrera (pp. 546-550). Coruña: Universidad de Coruña.

CARPENA, A. (2010). Desarrollo de las competencias emocionales en el marco escolar. CEE Participación Educativa, 15, 40-57.

CARPENA, A. (2013). Crecimiento emocional en el aula. En P. Darder, Aprender y educar con bienestar y empatía (pp. 69-102). Barcelona: Octaedro.

DIEKSTRA, R.E.W. (2008). Evaluación de los programas escolares universales de educación emocional y social y de habilidades para la vida. En C. Clouder (coord.), Educación Emocional y social. Análisis Internacional. Informe Fundación Marcelino Botín 2008 (pp.268-329). Santander: Fundación Marcelino Botín.

DURLAK, J. A. y WEISSBERG, R. P. (2010). Social and emotional learning programs that work. Better: Evidence-based Education, 2(2), 4-5.

DURLAK, J. A., WEISSBERG, R. P., DYMNICKI, A.B., TAYLOR, R.D., SCHELLINGER, K.B. (2011). The impact of enhancing students social and emotion learning: a meta-analysis of school-based universal interventions. Child Development, 82, 405-432.

EISENBERG, N., FABES, R. A. y SPINRAD, T. L. (2006). Prosocial development. En W. Damon y N. Eisenberg (Eds.), Handbook of child psychology, Vol. 3: Social, emotional and personality development (pp. 646-718). New York: John Wiley \& Sons.

EXTREMERA, N. y FERNÁNDEZ-BERROCAL, P. (2005). Inteligencia percibida y diferencias individuales en el metaconocimiento de los estados emocionales: una revisión de los estudios con el Trait Meta-Mood Scale. Ansiedad y Estrés, 12, 191-205.

EXTREMERA, N., FERNÁNDEZ-BERROCAL, P. (2013) La importancia de desarrollar la inteligencia emocional en el profesorado. Revista Iberoamericana de Educación, $33(8), 1-10$.

EXTREMERA, N., FERNÁNDEZ-BERROCAL, P., y SALOVEY, P. (2006). Spanish Version of the Mayer-Salovey-Caruso Emotional Intelligence Test (MSCEIT) Version 2.0: Reliabilities, Age, and Gender Differences. Psicothema, 18, 42-48.

FÉLIX-MATEO, V.; SORIANO-FERRER, M.; GODOY-MESAS, C. y MARTÍNEZ-RUIZ, I. (2007): Prevención de la violencia y promoción de la convivencia escolar en la Comunidad Valenciana. Plan PREVI. Aula Abierta, 36(1,2) pp 97-110.

FERNÁNDEZ-ABASCAL, E. (Coord.) (2008). Emociones positivas. Madrid: Pirámide. 
El desarrollo de las competencias emocionales en alumnado de secundaria: perfiles diferenciales en función del sexo

Alicia Peñalva-Vélez, José-Javier lópez-Goñi y Maria-Isabel García-Manso

FERNÁNDEZ-BERROCAL, P. y RUIZ-ARANDA, D. (2008). La inteligencia emocional en la educación. Revista Electrónica de Investigación Psicoeducativa, 15(6,2), 421-436.

GIMÉNEZ, M. y QUINTANILLA, L. (2009). Competencia "social", "competencia" emocional: una propuesta para intervenir en educación infantil. Infancia y Aprendizaje, 32(3), 359-373.

HARROD, N. R., y SCHEER, S. D. (2005). An exploration of adolescent emotional intelligence in relation to demographic characteristics. Adolescence, 40, 503-512.

LÓPEZ-GOÑI, J. J., FERNÁNDEZ-MONTALVO, J., y ARTEAGA, A. (2012). Addiction treatment dropout: Exploring patients' characteristics. American Journal on Addictions, 21(1), 78-85.

MARTínEZ, A.E., PIQUERAS, J.A. e INGLÉS, C. (2014). Relaciones entre Inteligencia Emocional y estrategias de afrontamiento ante el estrés. Extraído el 20 de enero de 2014, de http://reme.uji.es/articulos/numero37/article6/article6.pdf

MARTÍNEZ, A.E., PIQUERAS, J.A. y RAMOS, V. (2010). Inteligencia emocional en la salud física y mental. Electronic Journal of Research in Educational Psychology, 21(8, 2), 861-890.

MARTINS A., RAMALHO, N. y MORIN, E. (2010). A comprehensive meta-analysis of the relationship between Emotional Intelligence and health. Personality and Individual Differences, 49(6), 554-564.

MAYER, J. D., SALOVEY, P., y CARUSO, D. R. (2002). Mayer-Salovey-Caruso Emotional Intelligence Test (MSCEIT). Item Booklet, Toronto, Canada: MHS Publishers.

MESTRE, J.M., GUIL, R. y LIM, N. (2004). Inteligencia emocional: ¿a qué pueden deberse las diferencias respecto a la variable género? En E. Barberá, L. Mayor, M. Chóliz, E. Cantón, E. Carbonell, C. Candela y C. Gómez (eds.), Motivos, emociones y procesos representacionales: de la teoría a la práctica (313-324). Valencia: Fundación Universidad-Empresa de Valencia (ADEIT).

MOLERO, D. (2009). Competencias emocionales de los docentes durante su formación inicial. En Fundación Botín, Actas del Congreso Internacional de Inteligencia Emocional (CIIE - 2009) (pp.15-27). Santander: Universidad de Santander / Fundación Botín.

MOLERO, D., ORTEGA, F. y MORENO, M.R. (2010). Diferencias en la adquisición de competencias emocionales en función del género. Revista Electrónica de Investigación y Docencia (REID), 3, 165-172.

MURILLO, F.J. y HERNÁNDEZ, R. (2011). Efectos escolares de factores socio-afectivos. Un estudio multinivel para Iberoamérica. Revista de Investigación Educativa, 29(2), 407-427.

PALOMERA, R. (2008). Educando para la felicidad. En E. G. Fernández-Abascal (Coord.), Emociones positivas (pp.247-276). Madrid: Pirámide.

PALOMERA, R., GIL-OLARTE, P., y BRACKETT, M. A. (2006). ¿Se perciben con inteligencia emocional los docentes? Posibles consecuencias sobre la calidad educativa. Revista de Educación, 341, 687-703.

PENA, M. y EXTREMERA, N. (2012). Inteligencia emocional percibida en profesorado de Primaria y su relación con los niveles de burnout e ilusión por el trabajo (engagement). Revista de Educación, 359, 604-627.

PENA, M., EXTREMERA, N. y REY, L. (2011). El papel de la inteligencia emocional per- 
El desarrollo de las competencias emocionales en alumnado de secundaria: perfiles diferenciales en función del sexo Alicia Peñalva-Vélez, José-Javier López-Goñi y Maria-Isabel García-Manso

cibida en la resolución de problemas sociales en estudiantes adolescentes. REOP, 22(1), 69-79.

PENA, M. y REPETTO, E. (2008). Estado de la investigación en España sobre la Inteligencia Emocional en el ámbito educativo. Revista electrónica de Investigación Psicoeducativa. 6(2), 400-420.

PEÑALVA, A., LÓPEZ-GOÑII, J.J. y LANDA, N. (2013). Competencias emocionales del alumnado de Magisterio: posibles implicaciones profesionales. Revista de Educación, 362, 690-712.

PÉREZ-GONZÁLEZ, J. C. (2012). Revisión del aprendizaje social y emocional en el mundo. En R. Bisquerra (Coord.), ¿Cómo educar las emociones? La inteligencia emocional en la infancia y la adolescencia (pp. 56-69). Esplugues de Llobregat, Barcelona: Hospital Sant Joan de Déu.

PYHÄLTÖ, K., PIETARINEN, J. y SALMELA-ARO, K. (2011). Teacher-Working-Environment Fit as a Framework for Burnout Experienced by Finnish Teachers. Teaching and Teacher Education, 27, 1101-1110.

ROYO, M. (2013). Centros emocionalmente inteligentes. En P. Darder, Aprender y educar con bienestar y empatía (pp. 45-68). Barcelona: Octaedro.

SALGUERO, J.M. e IRUARRIZAGA, I. (2006). Relaciones entre inteligencia emocional percibida y emocionalidad negativa: ansiedad, ira y tristeza/depresión. Ansiedad y estrés, 12(2-3), 207-221.

SALMURRI, F. (2004). Libertad emocional: Estrategias para educar las emociones. Madrid. Circulo de Lectores.

SALMURRI, F. (2009). Índice de Bienestar Emocional (IGBE). No publicado.

SALMURRI, F. (2012). Avaluar les competències emocions a secundària. Escales per a la avaluació de les habilitats emocionals EHE-1. Barcelona: Fundació Propedagògic.

SALMURRI, F. (2013). Educación de las emociones, salud psicológica y centro educativo. En P. Darder, Aprender y educar con bienestar y empatía (pp. 23-44). Barcelona: Octaedro.

SALMURRI, F., SKOKNIC, V. (2005) Efectos conductuales de la educación emocional en alumnos de educación Básica. Revista psicológica de la Universidad de Chile, XIV, (1), 9-28.

SALOVEY, P., BEDELL, B., DETWEILER, J.B., y MAYER, J. D. (2000). Current directions in emotional intelligence research. En M. Lewis y J. M. Haviland-Jones (Eds.), Handbook of Emotions ( $2^{\text {nd }}$ ed.) (pp.504-520). New York: Guilford Press.

SÁNCHEZ, J. (2010). Sentido y función de las competencias básicas en educación. Revista Digital Sociedad de la Información, 24, 1-8.

SÁNCHEZ, J. (2011). La competencia emocional en la escuela: una propuesta de organización dimensional y criterial. ENSAYOS, Revista de la Facultad de Educación de Albacete, 25, 79-96.

SÁNCHEZ, M.T., FERNÁNDEZ-BERROCAL, P., MONTAÑÉS, J. y LATORRE, J.S. (2008). ¿Es la inteligencia emocional una cuestión de género? Socialización de las competencias emocionales en hombres y mujeres y sus implicaciones. Revista Electrónica de Investigación Psicoeducativa, 15(6,2), 455-474.

VALLÉS, A. (2008). La inteligencia emocional de los padres y de los hijos. Madrid: Pirá- 
El desarrollo de las competencias emocionales en alumnado de secundaria: perfiles diferenciales en función del sexo

Alicia Peñalva-Vélez, José-Javier López-Goñi y Maria-Isabel García-Manso

mide.

YOUNG, L. D. (2006). Parental influences on individual differences in emotional understanding. Dissertation Abstracts International: Section B: The Sciences and Engineering, 66(9), 5128B.

ZINS, J. E. (2004). Building academic success on social and emotional learning: what does the research says? New York: Teachers College Press.

\section{Agradecimientos}

Los autores agradecen a Ferrán Salmurri las facilidades dadas para el empleo de la Escala de Habilidades Sociales y del Índice General de Bienestar así como al profesorado y el alumnado del IES Donoso Cortés de Don Benito (Badajoz) sin cuya colaboración este proyecto no habría sido posible. 